To appear in the Astrophysical Journal

\title{
NGC 2442: Tidal Encounters and the Evolution of Spiral Galaxies
}

\author{
J. Christopher Mihos ${ }^{1,2}$ \\ Department of Physics and Astronomy, \\ Johns Hopkins University, Baltimore, MD 21218 \\ hos@pha.jhu.edu \\ and \\ Gregory D. Bothun \\ Department of Physics, \\ University of Oregon, Eugene, OR 97403 \\ nuts@moo.uoregon.edu
}

\begin{abstract}
Using imaging Fabry-Pérot data, we study the star-forming properties and kinematics of the nearby barred spiral galaxy NGC 2442. The $\mathrm{H} \alpha$ emission is very localized along the strong spiral arms of the galaxy, and shows a marked asymmetry between the sharp, well-defined northern tidal arm and the weaker southern arm. The $\mathrm{H} \alpha$ velocity field appears highly distorted, with a rapidly rotating nuclear component. We find evidence for strong non-circular motions along the northern arm, coincident with the pronounced dust lane and regions of intense star formation. The strong asymmetries, disturbed velocity field, and presence of a perturbed companion suggest that we are witnessing a strong kinematic response to a close interaction, which has redistributed the star formation activity throughout the disk of NGC 2442. Dynamical modeling of the NGC 2442 system supports this hypothesis, and suggests that the regions of strongest star formation are coincident with strong shocks occurring along the tidally perturbed northern arm. Despite this strong redistribution of the gas on small scales, this galaxy does not show a significant departure from the
\end{abstract}

\footnotetext{
${ }^{1}$ Hubble Fellow

${ }^{2}$ Visiting Astronomer, Cerro Tololo Inter-American Observatory. CTIO is operated by AURA, Inc. under contracts to the National Science Foundation.
} 
Tully-Fisher relation, nor does it appear to be experiencing any strong starburst. Moreover, our models predict that in a few x $10^{8}$ years, NGC 2442 will have recovered from this first tidal encounter and will experience another passage and ultimately a merger - in a few Gyr. This merger may provoke stronger, permanent changes in the structural properties of the galaxy, depending on the detailed response of the disk. Given the environment of many disk galaxies, this tidal encounter cycle seems likely to be a normal phase of disk galaxy evolution.

Subject headings: galaxies:evolution, galaxies:interactions, galaxies:individual (NGC 2442), galaxies:kinematics and dynamics, stars:formation 


\section{Introduction}

There is compelling morphological and photometric evidence that enhanced starforming activity in disk galaxies is a direct result of tidal interactions between galaxies. However, the physics of this strong causal relationship remain poorly understood. Although the strongest starbursts are generally found in the nuclei of interacting galaxies, some systems only show star formation in their outer disks, while others show no sign of elevated star formation rates at all. This variety suggests the local physics of gas dynamical response to perturbations varies considerably from interaction to interaction. While models of interaction-induced star formation can successfully explain strong central starbursts through bar-induced inflows (e.g., Noguchi 1988; Mihos, Richstone, \& Bothun 1992; Mihos \& Hernquist 1994, 1996), they have more difficulty exciting strong disk star formation. Moreover, induced star formation in interacting disks is often asymmetric and patchy in nature, suggesting that the local dynamical conditions in the galaxy are an important piece in the puzzle. To understand in detail how interactions influence star forming activity, we must simultaneously examine both the kinematics and the star forming properties of individual interacting systems.

The southern galaxy NGC 2442, shown in Figure 1, is a nearby ( $\mathrm{D}=16 \mathrm{Mpc}$ for $H_{0}=75$ $\mathrm{km} / \mathrm{s} / \mathrm{Mpc}$ ) peculiar barred spiral (SAB(s)bc, de Vaucouleurs et al. 1991 [RC3]) showing many signs indicative of an interaction. Along with the short central bar, ovally distorted body, and isophotal twists, the disturbed, asymmetric, and extended arms of the galaxy argue for a collisional origin. Table 1 summarizes the environment of NGC 2442. The galaxy has two nearby companions and is possibly a member of a loose group (Tully 1988). Because of its proximity, NGC 2442 offers a rare opportunity to investigate the kinematic and star forming properties of an interacting galaxy shortly after the initial collision. At 16 Mpc, $1^{\prime \prime}$ subtends $77 \mathrm{pc}$, affording a very detailed view into the response of a galaxy to a collisional encounter.

While a complete understanding of the dynamics of interacting galaxies is impossible when only morphological data exist, the combination of morphology and kinematic information can break the ambiguities arising from projection effects and yield a more accurate description of the dynamical state. Such an approach has been applied previously using kinematic information from slit spectra or HI data (e.g., Borne 1988; Borne et al. 1988; Balcells et al. 1989; Stanford \& Balcells 1991; Hibbard \& Mihos 1995). However, slit spectra do not provide full two-dimensional mapping of the ionized gas, while HI observations suffer from relatively low spatial resolution. The use of an imaging Fabry-Pérot spectrograph overcomes both these obstacles, providing two dimensional intensity and velocity maps of the ionized gas at $\sim 1^{\prime \prime}$ resolution (e.g., Tilanus \& Allen 1991; Vogel et al. 1993, Canzian 
et al. 1993; Mihos, Bothun, \& Richstone 1993). Using the morphology and velocity field in conjunction with numerical modeling, it is possible to reconstruct the dynamical history of interacting galaxies (e.g., Mihos et al. 1993) and address questions pertaining to the physical triggering mechanism for star-forming activity.

Numerical models of colliding galaxies demonstrate clearly that strong interactions drive rapid dissipation and inflow in the gaseous components of galaxies (e.g., Barnes \& Hernquist 1991, 1996; Mihos, Richstone, \& Bothun 1992; Mihos \& Hernquist 1994, 1996). This inflow is associated with the tidally-induced arms and bars in the galaxies, as gas is compressed along these features and driven inwards. In many galaxies, the radial motion along spiral features easily detectable: $\sim 30 \mathrm{~km} \mathrm{~s}^{-1}$ for M81 (Visser 1980), $\sim 50-70 \mathrm{~km} \mathrm{~s}^{-1}$ for UGC 2885 (Canzian et al. 1993), 60-90 $\mathrm{km} \mathrm{s}^{-1}$ for M51 (Vogel et al. 1988, Tilanus \& Allen 1991). The fact that M51, closely interacting with NGC 5195, shows the highest radial gas motions lends credence to the theoretical models of strong inflows in galaxy interactions and suggests such motions should be easily detectable in other interacting galaxies given the resolution of Fabry-Pérot data. The velocity field of the ionized gas in and around tidal features should therefore yield a great deal of information about the strength of the inflow and the physical conditions in the gas.

In this paper, we present imaging Fabry-Pérot data on NGC 2442 in order to explore the dynamical link between interactions, gaseous inflow, and star-forming activity. We find evidence for strong shocks and inflow along the galaxy's northern arm, accompanied by intense, localized star formation. Very little star formation is occurring in the southern tidal debris or along the bar itself, and in the inner kpc we detect a small, rapidly rotating ring of ionized gas. Based on these observations, we suggest a scenario in which NGC 2442 has recently (i.e. within a few hundred Myr) experienced a relatively strong interaction with AM 0738-692, driving the formation of the tidal arms. Dynamical modeling supports this scenario, and identifies the strong star forming sites along the arms as regions experiencing strong shocks and dissipation. The asymmetry observed in the star forming morphology of the galaxy seems to be related to the local dynamical conditions in the evolving tidal debris.

\section{Observations and Data Reduction}

NGC 2442 was observed in $\mathrm{H} \alpha$ with the Rutgers Imaging Fabry-Pérot on the CTIO 1.5-m telescope on the night of 1993 April 17. The Fabry-Pérot etalons have a bandpass of $\sim 2 \AA \mathrm{FWHM}$, giving an instrumental velocity dispersion of $\sim 50 \mathrm{~km} \mathrm{~s}^{-1}$. To isolate the $\mathrm{H} \alpha$ transmission order, a narrow band $(\sim 80 \AA) \mathrm{H} \alpha$ filter was used. With the Tek \#4 CCD, the pixel scale was $1.09^{\prime \prime}$ pixel $^{-1}$ and the circular field of view covered $\sim 7^{\prime}$ in diameter - 
enough to contain NGC 2442 itself, but none of its possible companions. NGC 2442 was scanned in $1 \AA$ steps from $6584 \AA-6604 \AA$, covering the full velocity width of the system. Observing conditions were not optimal, with seeing varying from $1.5^{\prime \prime}$ to $2^{\prime \prime}$ over the night and patchy cirrus present on occasion.

After standard bias-subtraction and flat fielding, the individual spectral images were shifted to a common centroid using foreground stars in the field. Sky subtraction proved more problematic, due to night sky emission lines in the spectral region scanned. Because of the radial spectral dispersion of the Fabry-Pérot, these night sky lines appear in the reddest nine frames as rings of diffuse emission, and had to be removed by subtracting model emission lines from each image. Once these sky lines were removed, a constant sky level could be obtained and subtracted from each frame. To account for changes in sky transparency, each image was normalized to a common transparency by performing photometry on the stars in the field and requiring those stars to have a flat continuum over the $20 \AA$ coverage of the observations. These normalization factors varied from 5 to $20 \%$, with typical uncertainties in the range of $5-10 \%$. To calibrate the wavelength solution of the instrument, neon arc lamp exposures were taken several times during the night.

After the data processing is complete, each pixel in the image has a 20 point spectrum associated with it, from which four pieces of data can be extracted: the continuum intensity, the integrated $\mathrm{H} \alpha$ flux, the radial velocity, and the velocity dispersion. These values are obtained by fitting a Voigt profile to the emission line profiles, binning pixels in regions of low flux to improve the signal and quality of fit. Typical errors are $\lesssim 10 \mathrm{~km} \mathrm{~s}^{-1}$ in velocity and $\lesssim 10 \%$ in $\mathrm{H} \alpha$ flux. Because of our relatively short exposure times in each band, the signal-to-noise level in the continuum is rather poor. To obtain a higher quality continuum image, a broad band V image was obtained by Bob Schommer using the $0.9 \mathrm{~m}$ telescope at CTIO on 1996 May 14. The reduced V-band image, consisting of 4 coadded 10 minute exposures, is shown in Figure 2.

\section{Morphology and Star Formation}

Figure $3 \mathrm{a}$ and $3 \mathrm{~b}$ show the reduced continuum and $\mathrm{H} \alpha$ intensity map for NGC 2442. The continuum maps (Figures 2 and 3a) reveal a number of NGC 2442's peculiarities. The bright, compact nucleus is clearly offset from the centroid of the outer isophotes by $\sim 20^{\prime \prime}$ $(\sim 1.5 \mathrm{kpc})$, suggesting a strong, differential distortion of the outer portions of the galaxy. The galaxy also shows severe isophotal twists - the major axis of the outer isophotes is aligned along a PA of $30^{\circ}$, while the major axis of the central regions twists from $40^{\circ}$ at $5^{\prime \prime}$ to $60^{\circ}$ at $20^{\prime \prime}$ (Sersic \& Donzelli 1993). The spiral arms are quite asymmetric: the northern 
arm is linear and well defined, extending $>20 \mathrm{kpc}$ from the main body, while the curved southern arm is much more diffuse and shorter, only $\sim 13 \mathrm{kpc}$ along its spine. The northern arm has a strong dust lane traced out over a large portion of the arm, whereas in the main body and southern arm the dust is more patchy in nature.

The star forming morphology also shows the asymmetry in the spiral arms. Table 2 summarizes the $\mathrm{H} \alpha$ photometry for NGC 2442. The bulk of the star formation in NGC 2442 is associated with the northern arm, and to a lesser extent with the inner portion of the southern arm. Star formation along these arms is very localized in giant HII regions, with little diffuse $\mathrm{H} \alpha$ observed. The differences in the star forming activity between the two arms are most evident in the outer portion of the arms. Using the spot at which each arm connects to the main body of the galaxy (roughly along a line of $30^{\circ} \mathrm{PA}$ ) to divide the arms into "inner" and "outer" regions, we find that the total amount of star formation in the inner regions of each arm is comparable, with each arm containing $\sim 22 \%$ of the total $\mathrm{H} \alpha$ flux from the galaxy. However, the outer portion of the arms are drastically different, with the outer northern arm providing $37 \%$ of the total $\mathrm{H} \alpha$ flux of the galaxy, compared to only $9 \%$ from the outer southern arm.

The nuclear regions show evidence for both emission from a central point source as well as a star-forming ring of radius $\sim 8^{\prime \prime}(620 \mathrm{pc}$; see Figure 4$)$. Studies into the nature of the central source in NGC 2442 have been inconclusive; using optical spectroscopy, Shobbrock (1966) classified it as as Seyfert, while Veron-Cetty \& Veron (1986) call it "Seyfert-like," but showing $\mathrm{H} \beta$ in absorption. The ring+point-source morphology of the $\mathrm{H} \alpha$ emission shown here suggests that the confusion is probably due to the fact that there is a combination of Seyfert (the point source) and starburst activity (the star-forming ring) occurring in the nucleus of NGC 2442. The lack of a strong HII region spectrum combined with the presence of $\mathrm{H} \beta$ in absorption suggests that the current starburst event in the central regions is waning.

Interestingly, although the spatial distribution of $\mathrm{H} \alpha$ appears to have been strongly altered by the interaction, the total $\mathrm{H} \alpha$ luminosity of NGC $2442\left(\log L_{H \alpha}=41.5\right.$; Ryder \& Dopita 1994) is quite normal for a non-interacting, luminous, late-type spiral (see e.g., Kennicutt et al. 1987). Similarly, NGC 2442 is not infrared luminous, with $\log L_{I R}=10.0$, and its infrared-to-blue luminosity ratio of $L_{I R} / L_{B}=0.56$ is typical of isolated spirals and an order of magnitude below interacting starburst galaxies (see Sanders \& Mirabel 1997). Following Kennicutt, Tamblyn, amd Congdon (1994), we can estimate the ratio of the

\footnotetext{
${ }^{3}$ Note that, because of the non-photometric observing conditions, we have derived only relative $\mathrm{H} \alpha$ fluxes across the galaxy, and used the calibration by Dopita \& Ryder (1994) for the photometric zeropoint.
} 
current SFR to the average SFR over the age of the disk using the $\mathrm{H} \alpha$ and optical (V) luminosity of the disk; for NGC 2442 we derive $b \equiv S F R /<S F R>_{\text {past }}=0.72$, similar to the median value of $\langle b\rangle=0.85$ for Sbc galaxies derived by Kennicutt et al. (1994). In short, while the galaxy has responded very strongly in its morphology, kinematics, and spatial distribution of star formation, its overall rate of star formation seems quite normal for its Hubble type. Evidently the local disturbances spawned by the interaction are more dramatic than the global response.

Finally, we point out a small, detached star forming region midway between the main body and the extended tip of the northern arm. This object, approximately $500 \mathrm{x} 300$ pc in size, has an $\mathrm{H} \alpha$ luminosity of $L_{H \alpha}=1 \times 10^{39} \mathrm{erg} \mathrm{s}^{-1}$, implying a star formation rate of $\sim 0.01 \mathrm{M}_{\odot} \mathrm{yr}^{-1}$, using the $L_{H \alpha} / \mathrm{SFR}$ conversion factor from Kennicutt (1983). It shows no sign of rotation in the $\mathrm{H} \alpha$ velocity map; however, its systemic velocity (1595 $\mathrm{km} \mathrm{s}^{-1}$ ) is intermediate to the velocity of the outer northern arm and the inner disk. It is unclear whether this object is a preexisting dwarf companion to NGC 2442, a massive HII region stripped from the inner disk, or new star forming region condensing from the tidally perturbed interarm ISM. However, its small size and $\mathrm{H} \alpha$ luminosity $(\sim 20 \%$ that of the Small Magellenic Cloud (Kennicutt et al. 1995)) argues against its status as a bona-fide dwarf companion (preexisting or newly formed). The relatively short lifetime of individual HII regions ( $\lesssim 10^{7}$ years; von Hippel \& Bothun 1990) also make it unlikely to be a stripped disk HII region. Models by Barnes \& Hernquist (1992) have demonstrated that adiabatically cooling gas in tidal debris can collapse and fragment into clumps. The region between the disk and tidal debris would be a suitable site for this process to occur, leading perhaps to star forming clumps such as this.

\section{4. $\mathrm{H} \alpha$ Kinematics}

Figure $3 \mathrm{c}$ and $3 \mathrm{~d}$ show the derived $\mathrm{H} \alpha$ velocity map and velocity dispersion map for NGC 2442. The FWHM of the velocity distribution is $400 \mathrm{~km} \mathrm{~s}^{-1}$; however, due to the strong distortion of the galaxy it is difficult to unambiguously assign an inclination and derive the rotation speed of the galaxy. Assuming the inner ring is circular, we can use its ellipticity $(b / a=0.45 \pm 0.06)$ to derive an inclination f of $i=69 \pm 4^{\circ}$, and an implied

\footnotetext{
${ }^{4}$ If the ring is intrinsically highly elliptical, this method will overestimate the inclination of the disk. We note that the inclination we derive is only somewhat higher than that reported in the literature $\left(i=24^{\circ}\right)$ from an analysis of the global axial ratio of the arms (e.g., Bajaja \& Martin 1985; Baumgart \& Peterson 1986). However, because of the obvious distortion in the outer isophotes, use of the global axial ratio to determine inclination is very suspect. Furthermore, the derived low inclination would yield an anomalously
} 
circular velocity for the system of $v_{\text {circ }}=225 \mathrm{~km} \mathrm{~s}^{-1}$. We can also use the velocity map of the inner ring and nucleus to solve for the systemic redshift; the median radial velocity for this region is $v_{\text {sys }}=1475 \pm 10 \mathrm{~km} \mathrm{~s}^{-1}$. This systemic velocity is greater by $40 \mathrm{~km} \mathrm{~s}^{-1}$ than the systemic velocity derived via HI (Bajaja \& Martin 1985) or CO (Bajaja et al. 1995) measurements; this discrepancy can easily be explained by the fact that the cold gas is preferentially found on the blue-shifted NE side of the galaxy (Bajaja et al. 1995). The mean redshift of the inner ionized ring is therefore a better estimate of the systemic velocity of NGC 2442.

Using the derived $\mathrm{H} \alpha$ velocity map, we attempt to construct a rotation curve for NGC 2442 in the manner described by Schommer et al. (1993). Using the inferred inclination $i$ and major axis PA, we can derive the circular velocity as a function of radius in concentric annuli, shown in Figure 5. The derived rotation curves are sensitive to the choice of kinematic center, which is ill-defined in the case of NGC 2442. Two possibilities are examined: first, that the kinematic center is marked by the nucleus, and second, that the kinematic center is defined by the centroid of the disk isophotes. As noted previously, the nucleus is offset from the isophotal center of the galaxy by $20^{\prime \prime}$, and because of the lack of $\mathrm{H} \alpha$ emission from $\mathrm{R}=10^{\prime \prime}-70^{\prime \prime}$, it is difficult to discern kinematically which option is more physically reasonable. With nucleus-centering, the rotation curve shows a rapid rise, then falls with radius; with isophotal-centering, the rotation curve is rising over much of the disk. Further complicating the rotation curve derivation is the fact that the $\mathrm{H} \alpha$ emission is preferentially found along the tidally perturbed spiral arms, where non-circular motions should be highest. Tests using the numerical models described in $\S 6$ indicate that the shape of the derived rotation curve is sensitive to the azimuthal portion of the disk sampled by the $\mathrm{H} \alpha$ emission. This kinematic bias, coupled with the sparseness of the $\mathrm{H} \alpha$ data and the uncertainty in the kinematic center, does not allow for a physically meaningful constraint on either the mass distribution or the global kinematics of NGC 2442; as a result we focus on the two-dimensional velocity map rather than the rotation curve in our discussion of kinematics and model matching below.

One thing is clear from the rotation curve, however: the ionized gas in the nuclear region shows a steep rise in rotation velocity, reaching a maximum of $v \sin i=220 \pm 10 \mathrm{~km}$ $\mathrm{s}^{-1}$ only $8^{\prime \prime}$ (500 pc) from the nucleus. Unlike the outer body, the velocity field of the inner ring does show a simple spider-diagram indicating circular motion and we derive a mass interior to the ring of $M_{n u c}=R v^{2} / G=8.4 \pm 1.5 \times 10^{9} \mathrm{M}_{\odot}$. The rapidly rising rotation

large rotation velocity of $v_{c i r c}=490 \mathrm{~km} \mathrm{~s}^{-1}$. More recently, Ryder and Dopita determined the inclination from the axial ratio of the inner disk and found $i=60^{\circ}$, similar to the value we derive from the starburst ring. 
curve and large mass interior to $500 \mathrm{pc}$ is consistent with the bright, compact nucleus seen in the continuum map. The velocity dispersion of the ionized gas in the ring is rather high, $\sim 50-60 \mathrm{~km} \mathrm{~s}^{-1}$, while in the central source itself the velocity dispersion reaches as high as $125 \mathrm{~km} \mathrm{~s}^{-1}$.

Striking evidence for strong noncircular motions in NGC 2442 comes from the kinematic patterns evident in the two-dimensional velocity map (Figure 3c). In the main body of the galaxy these velocity patterns can also be seen in the CO map of Bajaja et al. (1995), albeit at much lower spatial resolution. In the classic "spider diagram" for a disk in circular rotation, isovelocity contours fan out in a V-shaped pattern along the major axis. In such a velocity field, cuts parallel to the minor axis will show velocity symmetry across the major axis. NGC 2442 shows no such behavior; rather, the NW and SE portions of the disk show skewed velocity patterns. On the SW side of the disk, a cut parallel to the minor axis at $\mathrm{R}=75^{\prime \prime}$ (5 kpc), shows a rather large velocity difference of $\sim 120 \mathrm{~km} \mathrm{~s}^{-1}$ between the NW and SE extrema of the cut. A similar cut along the NE side of the disk shows a velocity difference of $\sim 150 \mathrm{~km} \mathrm{~s}^{-1}$ across the cut. In essence, rather than being symmetric around the minor axis $\left(\mathrm{PA}=120^{\circ}\right)$, the isovelocity contours in the inner disk run nearly N-S, along a PA of $0^{\circ}$. The fact that the isovelocity contours are distorted along the spiral arms is a clear indication of strong non-circular motion induced by the arms. Further out in the tidal arms, the isovelocity contours twist again, following a PA of $155^{\circ}$.

The most curious portion of the velocity field lies in the NE side of the disk, where the northern tidal arm connects to the galaxy. The very strong dust lane cuts through this region, and the strongest sites of star formation are located here as well. Running parallel to the dust lane, but offset to the inside by 4 " (250 pc), is a low velocity "trough" in the velocity field of the arm (see Figure 6). In this portion of the arm, the isovelocity contours run parallel to the arm, dropping from $1280-1290 \mathrm{~km} \mathrm{~s}^{-1}$ on the inside of the arm to 1230 - $1240 \mathrm{~km} \mathrm{~s}^{-1}$ in the trough, before rising again to $1280-1290 \mathrm{~km} \mathrm{~s}^{-1}$ on the outside of the arm. Along this trough, the velocity dispersion in the gas reaches its highest (non-nuclear) values: $75-85 \mathrm{~km} \mathrm{~s}^{-1}$, compared to more typical values of $30-40 \mathrm{~km} \mathrm{~s}^{-1}$ in other regions of the galaxy and tidal arms. The velocity dispersions are increased from the point where the arm enters the disk, and continue along the arm until the point where the arm turns back towards the inner regions of the disk. The strongest sites of $\mathrm{H} \alpha$ emission occur on the outside of the dust lane; however, it is unclear whether this reflects an offset in the physical location of the star formation, or merely strong obscuration of the $\mathrm{H} \alpha$ line in the dust lane.

Finally we note the velocity structure along the outer portion of the northern arm. This tidal arm shows a modest velocity gradient along the spine of the arm, such that regions further along the tail are subsequently more redshifted (by $200 \mathrm{~km} \mathrm{~s}^{-1}$ over the 
$20 \mathrm{kpc}$ span of the arm). However, the isovelocity contours run largely parallel to the tidal arm, in the sense of a smooth gradient (rather than velocity trough) across the arm. Perpendicular cuts through the arm reveal velocity differences of $\sim 100 \mathrm{~km} \mathrm{~s}^{-1}$ across the kiloparsec breadth of the arm, similar to the gradients observed in the tidal arms of the interacting galaxy NGC 6872 (Mihos et al. 1993).

The combined information in the morphology and velocity maps presented in Figures 2, 3, and 6 suggests the following scenario along the northern edge of NGC 2442's disk. The tidal interaction has drawn out the northern arm from the main body of the galaxy, compressing the gas along the spine of the arm. As shown by numerical modeling of tidal features (e.g., Hernquist \& Spergel 1992; Hibbard \& Mihos 1995; see also §6 below), material in the tidal arm now falls back inwards towards the main body. As gas returns to the disk, it is shocked and compressed where the arm and disk intersect. A similar fate may hold for gas on the $\mathrm{W}$ side of the disk, rotating into this contact point. The high velocity dispersion where gas enters the shock (upstream from the dust lane) attests to the turbulent motion in the preshock region. As the gas is compressed along the shock, it dissipates energy and begins to flow inward, decoupling from the motion of the surrounding arm (and thus producing the velocity trough). Downstream from the shock, star formation occurs in the cooling postshock gas, as evidenced by the strong $\mathrm{H} \alpha$ emission along the outside ridge of the dust lane.

While these local effects are dramatic it is important to verify if interactions like these are so severe to nullify including this galaxy in any kind of Tully-Fisher (TF) analysis. Recall that an imaging study of this galaxy might not necessarily flag it as being strongly interacting as there are currently no obvious close, luminous companions. We can place NGC 2442 on the TF relation by using its observed $\mathrm{R}$ magnitude of $R=9.71 \pm 0.09$ (RC3) and a distance modulus of $31.0 \pm 0.3$ (Sersic \& Donzelli 1993), and correcting for galactic absorption via Burstein \& Heiles (1984) and internal absorption via Tully \& Fouque (1985), after which we derive an absolute $\mathrm{R}$ magnitude of $M_{R}=-22.1 \pm 0.3$. Using the HI velocity width from Bajaja \& Martin (1985), and correcting for inclination, we can now put NGC 2442 on the R-band TF relationship derived by Pierce \& Tully (1992). Despite the uncertainties in the inclination and internal absorption, Figure 7 shows that NGC 2442 lies essentially on the TF relation defined by the local calibrators. Although the detailed kinematics of the galaxy have been significantly affected by the interaction, surprisingly, its overall dynamical behavior has not. Our results suggest that studies of the TF relation at higher redshift will not be compromised by effects due to galaxy interactions, save for specific cases where the interaction is very strong (i.e. a merger). 


\section{Who's to Blame?}

The disturbed morphology and velocity field of NGC 2442 suggest strongly that the galaxy was involved an encounter in the not-too-distant past. NGC 2442 may be a member of a loose group (Tully 1988), and several nearby neighbors do exist; in particular, the E0 galaxy NGC 2434 and the small SB0/a AM 0738-692. Both galaxies lie at the same redshift as NGC 2442 (see Table 1). AM 0738-692 is the closer of the two companions, at a projected separation of $50 \mathrm{kpc}$; however, it is fainter by 2.2 magnitudes in $\mathrm{R}$ than NGC 2442, suggesting a rather large mass ratio (assuming similar M/L's for the galaxies) of 7.5:1. Although twice as far away as AM 0738-692, NGC 2434 is brighter by almost a magnitude in R, and the implied mass ratio between NGC 2442 and NGC 2434 is more hefty $3: 1$. No other prominent galaxies lie with a projected distance of $400 \mathrm{kpc}$ of NGC 2442 (Table 1).

Despite its low mass, we favor AM 0738-692 as the perturber due to its disturbed morphology. Figure 8 shows the morphology of AM 0738-692 taken from the Digital Sky Survey. The galaxy has a very asymmetric light distribution, with highly twisted outer isophotes. In fact, the outer arms look suspiciously like the broad tidal tails which would develop in a galaxy with large $\sigma / v$. A spectrum of AM 0738-692 reveals a pure stellar component (i.e. no emission lines indicative of star formation) with a velocity dispersion of of $\sigma \sim 120 \mathrm{~km} \mathrm{~s}^{-1}$ (G. Aldering, private communication), consistent with the SB0/a classification of the galaxy, and the lack of strong tidal arms normally spawned in interactions involving cold stellar disks. Because of the relatively high inferred mass ratio for the galaxy pair, the collision must have happened quite recently - within the past few galactic rotation periods - for NGC 2442 to still show such strong asymmetries and velocity distortions.

\section{Dynamical Modeling}

In order to gain more insight into the dynamical history of NGC 2442, we simulate a NGC 2442-type encounter, following the evolution of both the stellar and gaseous components of the galaxies. Recent attempts to model specific interacting systems have yielded reasonable success (see, e.g., Barnes 1988; Mihos et al. 1993; Hibbard \& Mihos 1995; Hibbard \& Barnes 1996), although the derived solutions depend critically on the chosen dark matter distribution in the galaxies (Mihos, Dubinski, \& Hernquist 1996). This result suggests that any given model may suffer from a severe lack of uniqueness due to the poorly constrained dark matter distribution. Rather than attempting an exhaustive survey of orbital configurations and galaxy models to recreate the system in minute detail, we choose 
to focus on an encounter which captures the important features of the NGC 2442 system.

The initial galaxy models are constructed using the technique described by Hernquist (1993), wherein the primary galaxy consists of a spherical dark halo, an exponential disk of both stars and gas, and a compact central bulge. We use a system of units in which the gravitational constant $G=1$, the disk scale length $h=1$, and the disk mass $M_{d}=1$. The disk is populated with both star and gas particles out to a maximum radius of $R_{d}=15$. The halo is modeled as a "truncated" isothermal sphere of mass $M_{h}=5.8$, core radius $\gamma=1$, exponential cutoff radius $r_{t}=10$, and maximum radius $R_{h}=30$. The central bulge follows a flattened Hernquist (1990) profile, with scale length $a=0.14$, flattening $c / a=0.5$ and total mass $M_{b}=1 / 9$. The resulting rotation curve for the model is similar to the "universal" rotation curve for bright spirals defined by Persic, Salucci, \& Stel (1996). The gas comprises $10 \%$ of the disk mass, and is distributed in the same exponential profile defined by the disk stars. For expediency, an isothermal equation of state is chosen for the gas, with temperature $T_{\text {gas }}=10^{4} \mathrm{~K}$. The companion galaxy is modeled as a gas-free spherical Hernquist (1990) model with total mass $M_{c}=0.90$. The companion mass was chosen to give a mass ratio between the galaxies of 7.5 , similar to the observed $\mathrm{R}$ band luminosity ratio between NGC 2442 and AM 0738-692. For the final models shown here, a total of 102,400 particles were used: 32,768 each in the stellar disk and dark halo; 4,096 in the central bulge; 16,384 in the companion galaxy; and 16,384 SPH particles to follow the evolution of the disk gas. The simulation was evolved using a combined N-body/smoothed particle hydrodynamics code (TREESPH; see Hernquist \& Katz 1989), and run on the Cray C90 at the San Diego Supercomputing Center.

Having selected the galaxy models, the remaining parameters describing the system are the orbital energy, geometry, collision time, and viewing angle. We choose a parabolic (zero-energy) orbit for the encounter; hyperbolic encounters are unable to excite strong tidal features at this mass ratio, while a tightly bound orbit is unlikely given the (relatively) large current separation of the galaxy pair. In order to gain some constraint on the orbital parameters, we ran a set of pure $N$-body models (i.e. no gas), varying perigalactic distance $\left(R_{p}\right)$, orbital inclination $(i)$, and argument of periapse $(\omega$; see e.g., Toomre \& Toomre 1972). Not surprisingly, degeneracies exist between the models when viewed from different angles and at differing times. Nonetheless, some constraints were clear. No model could successfully reproduce the strong coherent NW tidal arm at times much later than a rotation period after the initial collision. Regardless of orbital inclination, no model was able to torque disk material significantly out of the disk plane. Finally, very distant encounters had trouble exciting tidal features, while in close encounters the companion galaxy remains too close to the primary, when compared to the NGC 2442 system. Our best "approximate match" model uses $R_{p}=5.0, i=\omega=45^{\circ}$, and is observed at 20 time 
units ( $\sim 1.5$ half-mass rotation periods) past perigalacticon.

Figure 9 shows the evolution of the NGC 2442 model, observed in the plane of the sky. Only the stellar disk and companion are shown in the figure. The galaxies reach perigalacticon at $\mathrm{T}=25$, and the current viewing time is $\mathrm{T}=46$. To convert from model units to physical units, we scale the model in size and velocity such that the disk size (measured along the major axis PA from arm to arm) and FWHM of the velocity distribution match the observed values. With this scaling, unit length is $3 \mathrm{kpc}$, unit velocity is $265 \mathrm{~km} \mathrm{~s}^{-1}$, unit mass is $4.8 \times 10^{10} \mathrm{M}_{\odot}$, and unit time is $11.5 \mathrm{Myr}$. The scaled time since collision is thus $230 \mathrm{Myr}$; at this time, the galaxies have reached a projected separation of $48 \mathrm{kpc}$ and the companion has line-of-sight velocity of $+25 \mathrm{~km} \mathrm{~s}^{-1}$ (with respect to NGC 2442), comparable to the observed values of $50 \mathrm{kpc}$ and $+54 \pm 53 \mathrm{~km} \mathrm{~s}^{-1}$. The inclination of the galaxy to the sky plane is $\sim 60^{\circ}$. At the observed time, the model is not a perfect match in particular, the pitch angle of the NW arm is smaller in the model, and the strength of the southern arm is somewhat stronger. Nonetheless, these discrepancies are fairly minor, and this simulation should provide a reasonable description of the dynamical state of the NGC2442 system.

Even at this relatively large mass ratio, the encounter has no trouble exciting strong tidal features like those observed in NGC 2442, yet the companion still has managed to reach the necessary separation of $50 \mathrm{kpc}$. As the companion passes by the primary, it draws material out in a rather diffuse, illusory bridge. In particular, note time $\mathrm{T}=40$, where this "bridge" consists of a spray of material drawn from the nearside of the disk, while the strong tail on the opposite side is more coherent in structure, flaring only near its end. As the model evolves towards the current time, material falls back towards the disk from both tidal features, compressing along the spiral features in the disk. The gas densities are highest along the spiral arms and in the nuclear region (see Figure 10). In addition, the NW tidal arm is well separated from the main disk, while the southern tidal debris is more diffuse, with a continuous spray of material from the disk out to the outer edge of the SE arm.

With the kinematic information provided in the model, we can look at the physical conditions in the gas along the tidal arm, the analogue of NGC 2442's strong NW arm. Figure 11 shows the velocity structure in the model, with vectors showing the particles' projected tangential velocity in each frame. The strong streaming along the spiral arms is apparent; as material enters the arms, it shocks and begins to flow inwards along the arm. In projected radial velocity, the same skewed velocity field is seen in the model as in NGC 2442. Inside the inner scale length of the disk, the arms weaken in strength due to the stabilizing influence of the bulge and the velocity patterns become more circular; however, 
some net inflow still occurs.

Focusing on the kinematic structure in the NW arm, we see that the point at which the arm connects into the main body is in fact dominated by orbit crossing in the gas, as material from the disk streams into the tidal arm and dissipates energy. In NGC 2442, this point is characterized by strong star formation and the low velocity trough. The trough and the streaming along the outer arm suggested in the observed velocity of NGC 2442 are visible in a line-of-sight radial velocity map of the model, but to a lesser degree than observed in NGC 2442. Most likely this is a resolution effect - while the global kinematic structure of the tidal arm is well reproduced, the hydrodynamic smoothing length of the gas particles is comparable to the width of the arm, making substructure within the arm hard to resolve. Alternatively, there is some indication that the tidal perturbation on the galaxy is even greater than suggested by the model - the offset nucleus, the wide pitch angle of the NW arm - which may result in a stronger decoupling of gas velocities along the tidal arm.

On the whole, our model does a reasonable job of matching both the morphology and kinematics of the NGC 2442/AM 0738-692 pair. In particular, we are able to match the asymmetry in the tidal arms, the relative position and velocity of AM 0738-692, and the skewed velocity field of NGC 2442. The model also reproduces the kinematic features along the northern side of the disk associated with shocks and dissipation in the gas returning from the NW tidal arm. Some discrepancies remain, however, including the too-prominent southern arm, the lack of an offset nucleus, and the smooth distribution of gas in the inner disk. Further improvements on the model are possible, and could take the form of differing dark matter distributions (to alter the disk response), orbit geometries (to enhance the asymmetry of the tidal arms), and/or gas distributions in the pre-encounter disk of NGC 2442. Nonetheless, we are encouraged by the quality of the model, given the minimal amount of "tuning" of parameters in our model matching effort.

What is the future fate of our modeled encounter, and, by extension, the NGC 2442/AM 0738-692 pair? After another disk rotation period ( 350 Myr after the collision), much of the material currently in the extended tidal debris has begun to fall back towards the disk and tidal features are becoming quite diffuse. In the disk, a strong grand-design spiral structure persists, but after 500 Myr distinct signatures of the tidal encounter are hard to identify. Although warped and somewhat heated by the tidal encounter, the disk galaxy appears largely axisymmetric, with no strong tidal distortions. The action is not yet finished, however; orbital energy has been transferred to internal motions of the galaxies, which find themselves on a loosely bound orbit. We have followed the subsequent evolution of the galaxies and find that after reaching a maximum separation of $\sim 100$ kpc, AM 0738-692 begins to fall back towards NGC 2442, with a second close passage 
$\left(r_{\text {peri }} \sim 20 \mathrm{kpc}\right)$ occurring in another 2 Gyr. After this, the orbital decay is quite rapid, and following a third close encounter the galaxies ultimately merge after a total elapsed time of 3 Gyr. This merger may be quite destructive to the disk of NGC 2442. In our model, the companion survives the intermediate passages with most of its mass intact, so that it has a strong impact when it reaches the disk of NGC 2442. As a result, the thin, cold disk is largely destroyed by the merger. The merged object is still highly flattened, however, and shows significant rotation. Rather than resembling the very elliptical-like remnants formed in major mergers of comparable mass galaxies (e.g., Barnes 1992; Barnes \& Hernquist 1996; Mihos \& Hernquist 1996), this remnant may be more akin to S0 galaxies, with large bulge:disk ratios, diffuse disks, and high $\sigma / v$.

We caution, however, that the final results of the model are strongly dependent on both the distribution of dark matter around the galaxies and the internal structure of the companion galaxy. Unfortunately, neither of these values are well constrained. Because the density and dark matter content of the galaxies determines the degree to which the companion is stripped of mass, a lower density companion, coupled with an extended distribution of dark matter around NGC 2442, could sufficiently strip the companion of

mass, extending the merging timescale and reducing the impact on the disk of NGC 2442. While the final outcome of such intermediate mass mergers is crucial to understand, a proper treatment of the problem is beyond the scope of the models presented here.

\section{Summary}

Using Fabry-Pérot mapping of the morphology and kinematics of NGC 2442 in conjunction with a representative numerical model of the system, we have explored the triggering of star forming activity by a close interaction. Our results indicate that in the recent past, $\sim 150-250$ Myr ago, NGC 2442 and AM 0738-692 experienced a close encounter, resulting in the tidal distortions currently observed in both galaxies. The distorted isophotes and velocity patterns throughout the disk of NGC 2442 attest to the damage done by even this relatively high mass ratio encounter. Material on the side of the disk closest to the companion experienced significant tidal shear, shredding much of the coherent tidal structure. Conversely, material on the far side of the disk was subject to a more coherent tidal compression, resulting in the formation of the dramatic northern tidal arm. As gas returns to the disk along this arm, it is being shocked and recompressed, forming a ridge of intense star formation and radial inflow along the NGC 2442's northern edge.

The asymmetric pattern of star formation in NGC 2442 is a reflection of the differing 
physical conditions in the gas on either side of the galaxy. On the northern side of the galaxy, the gas is undergoing strong shocking and dissipation along the tidally induced arm, resulting in the vigorous, localized star formation. The extended tidal arm on the south side of the galaxy is much more diffuse, and the gas is not as strongly compressed along this feature. This kinematic structure is a reflection of the formation history of the tidal arms. Material close to the companion at perigalacticon experiences a very strong tidal shear, dragging material out into a transient tidal bridge which rapidly disperses (e.g., Toomre \& Toomre 1972; Mihos et al. 1992, 1993). In contrast, material on the opposite side of the disk feels a gentler - but still effective - tidal field, resulting in a more coherent tidal tail (or, in a high mass ratio encounter such as this, tidal arm). In this scenario, the south side of NGC 2442's disk was the closest point to the passing companion; in the subsequent time since the encounter, the outer disk has rotated significantly, now presenting the NE side closest to AM 0738-692in projection.

The subsequent evolution of the NGC 2442 system is unclear. It is likely that the interaction will have extracted sufficient energy to bind the galaxies, and in our model the galaxies will merge in $\sim 3 \mathrm{Gyr}$. In principle, this ultimate merger may be violent enough to lead to a transformation of NGC 2442 from Sbc to Sa or even S0, depending on the detailed structure of the merging galaxies. In the meantime, NGC 2442 will have many rotation periods to resettle into a relatively normal configuration. While the local gas dynamical response to these first encounters can be dramatic, the collisions don't seem to significantly alter the basic structure and global properties of large disk galaxies, until the orbit decays sufficiently to lead to a merger. We are most probably catching NGC 2442 in a rather transient but common phase of disk galaxy evolution - recovering from a recent collision, and awaiting another yet to come.

We thank Bob Schommer for obtaining the V band image of NGC 2442, and Greg Aldering for the spectrum of AM 0738-692. We also thank Ted Williams for observing support, Ben Weiner and Charles Beauvais for helpful suggestions during the data reduction, Stuart Ryder for providing the $H \alpha$ flux calibration, and Gerhardt Meurer for a helpful review of the manuscript. Marc Balcells provided a thorough referee's report which improved the presentation of the paper. J.C.M. is supported by NASA through a Hubble Fellowship grant \# HF-01074.01-94A awarded by the Space Telescope Science Institute, which is operated by the Association of University for Research in Astronomy, Inc., for NASA under contract NAS 5-26555. Partial computing support for this project was provided by the San Diego Supercomputer Center.

This research has also made use of the NASA/IPAC Extragalactic Database (NED) which is operated by the Jet Propulsion Laboratory, California Institute of Technology, 
under contract with the National Aeronautics and Space Administration. The Digitized Sky Survey images are based on photographic data obtained using The UK Schmidt Telescope. The UK Schmidt Telescope was operated by the Royal Observatory Edinburgh, with funding from the UK Science and Engineering Research Council, until 1988 June, and thereafter by the Anglo-Australian Observatory. Original plate material is copyright (c) the Royal Observatory Edinburgh and the Anglo-Australian Observatory. The plates were processed into the present compressed digital form with their permission. The Digitized Sky Survey was produced at the Space Telescope Science Institute under US Government grant NAG W-2166. 


\section{REFERENCES}

Bajaja, E., Wielebinski, R., Reuter, H.-P., Harnett, J.I., \& Hummel, E. 1995, A\&AS, 114, 147

Bajaja, E., \& Martin, C.M. 1985, AJ, 90, 1783

Balcells, M., Borne, K.D., Hoessel, J.G. 1989, ApJ, 336, 655

Barnes, J.E. 1988, ApJ, 331, 699

Barnes, J.E. 1992, ApJ, 393, 484

Barnes, J.E., \& Hernquist, L.E. 1991, ApJ, 370, L65

Barnes, J.E., \& Hernquist, L.E. 1992, Nature, 360, 715

Barnes, J.E., \& Hernquist, L.E. 1996, ApJ, 471, 115

Baumgart, C.W., \& Peterson, C.J. 1986, PASP, 9856

Borne, K.D. 1988, ApJ, 330, 61

Borne, K.D., Balcells, M., \& Hoessel, J.G. 1988, ApJ, 333, 567

Burstein, D., \& Heiles, C. 1984, ApJS, 54, 33

Canzian, B., Allen, R.J., Tilanus, R.P.J. 1993, ApJ, 406, 457

Dahlem, M., Bomans, D.J., \& Will, J.-M. 1994, ApJ, 432, 590

de Vaucouleurs, G., de Vaucouleurs, A., Corwin, H.G., Buta, R.J., Paturel, G., \& Fouque, P. 1991, Third Reference Catalogue of Bright Galaxies, Springer-Verlag (RC3)

Dopita, M.A., \& Ryder, S.D. 1994, ApJ, 430, 163

Hernquist, L. 1990, ApJ, 356, 359

Hernquist, L. 1993, ApJS, 416, 9

Hernquist, L., \& Katz, N. 1989, ApJS, 70, 419

Hernquist, L. \& Spergel, D.N. 1992, ApJ, 399, L117

Hibbard, J.E., \& Mihos, J.C. 1995, AJ, 110, 140

Hibbard, J.E., \& Barnes, J.E. 1996, in preparation

Kennicutt, R.C. 1983, ApJ, 272, 54

Kennicutt, R.C., Bresolin, F., Bomans, D.J., Bothun, G.D., \& Thompson, I.B. 1995, AJ, 109,594

Kennicutt, R.C., Tamblyn, P., \& Congdon, C.E. 1994, ApJ, 435, 22

Mathewson, D.S., Ford, V.L., \& Buchhorn, M. 1992, ApJS, 81, 413 
Mihos, J.C., Bothun, G.D., \& Richstone, D.O. 1993, ApJ, 418, 82

Mihos, J.C., Dubinski, J., \& Hernquist, L. 1996, in preparation

Mihos, J.C., Richstone, D.O., Bothun, G.D. 1992, ApJ, 400, 153

Mihos, J.C., \& Hernquist, L. 1994, ApJ, 431, L9

Mihos, J.C., \& Hernquist, L.E. 1996, ApJ, 464, 641

Persic, M., Salucci, P., \& Stel, F. 1996, MNRAS, 281, 27

Pierce, M.J., \& Tully, R.B. 1992 ApJ, 387, 47

Ryder, S.D., \& Dopita, M.A. 1994, ApJ, 430, 142

Sanders, D.B., \& Mirabel, I.F. 1997, ARA\&A, in press

Schommer, R.A., Bothun, G.D., Williams, T.B., \& Mould, J.R. 1993, AJ, 105, 97

Sérsic, J.L. \& Donzelli, C. 1993, A\&AS, 98, 21

Stanford, S.A., \& Balcells, M. 1991, ApJ, 370, 118

Tilanus, R.P.J., \& Allen, R.J. 1993, A\&A, 274, 707

Toomre, A., \& Toomre, J. 1972, ApJ, 178, 623

Tully, R.B., \& Fouque, P. 1985, ApJS, 58, 67

Véron-Cetty, M.-P., \& Véron, P. 1986, A\&AS, 66, 335

Visser, H.C.D. 1980, A\&A, 88, 149

Vogel, S.N., Rand, R.J., Gruendl, R.A., Teuben, P.J. 1993, PASP, 105, 666

von Hippel, T., \& Bothun, G. 1990, AJ, 100, 403 
Fig. 1.- A Digitized Sky Survey image, centered on NGC 2442, also showing NGC 2434 (upper right) and AM 0738-692 (left). The image measures $30^{\prime}$ on a side. North is up; east is to the left.

Fig. 2.- Broad band V image of NGC 2442. North is up; east is to the left.

Fig. 3.- Reduced Fabry-Pérot maps of NGC 2442. a) Continuum map, b) H $\alpha$ flux map, c) velocity map, b) velocity dispersion map. In the velocity map, light shading represents blueshift, while dark shading represents redshift (relative to systemic). In the velocity dispersion map, dark shading indicates high velocity dispersion.

Fig. 4.- Close up image of the $\mathrm{H} \alpha$ intensity map in the inner disk.

Fig. 5.- Rotation curves derived from $\mathrm{H} \alpha$ velocity maps. The left panel shows the rotation curve derived with the nucleus as the kinematic center of the galaxy, while the right panel shows the rotation curve derived assuming the kinematic center is defined by the centroid of the disk isophotes.

Fig. 6.- Close up image of the $\mathrm{H} \alpha$ velocity and velocity dispersion maps in the northern arm.

Fig. 7.- NGC 2442 on the R-band Tully-Fisher relationship of Pierce \& Tully (1992). The dashed lines represent the $\pm 1 \sigma$ dispersion in the relationship. Errorbars on the NGC 2442 point represent a \pm 0.3 magnitude error in the distance modulus and a $\pm 4^{\circ}$ error in inclination.

Fig. 8.- A close-up from the Digitized Sky Survey image, centered on AM 0738-692. Note the distorted fan-like arms.

Fig. 9.- Evolution of the NGC 2442 model, viewed in the "sky plane." Only particles from the stellar disk and companion are shown. The frames measure 20 length units on a side, and time is shown in the upper right.

Fig. 10. - Grayscale representation of the gas distribution in the NGC 2442 model at the best match time. 
Fig. 11.- The velocity field of the gas in the NGC 2442 model at the best match time. The vectors represent the velocity vectors projected onto the plane of the figure. The left side shows the model observed in the sky plane; the right side shows the model observed in the disk plane. 
Table 1: Galaxies in the NGC 2442 Field

\begin{tabular}{cccc}
\hline Galaxy & $R_{T}{ }^{\mathrm{a}}$ & $\begin{array}{c}c z \\
\left(\mathrm{~km} \mathrm{~s}^{-1}\right)\end{array}$ & $\begin{array}{c}R_{\text {proj }} \\
(\mathrm{kpc})\end{array}$ \\
\hline NGC 2442 & $9.71 \pm 0.09$ & $1475 \pm 10^{\mathrm{b}}$ & - \\
AM 0738-692 & $11.91 \pm 0.09$ & $1529 \pm 53^{\mathrm{c}}$ & 50 \\
NGC 2434 & $10.84 \pm 0.09$ & $1390 \pm 27^{\mathrm{a}}$ & 84 \\
NGC 2397 & $11.57 \pm 0.09$ & $1363 \pm 10^{\mathrm{d}}$ & 420 \\
\hline
\end{tabular}

${ }^{a}$ from the RC3.

${ }^{b}$ this work.

${ }^{c} \mathrm{G}$. Aldering, private communication.

${ }^{d}$ Mathewson, Ford, \& Buchhorn 1992.

Table 2: $\mathrm{H} \alpha$ Photometry of NGC 2442

\begin{tabular}{ccc}
\hline Region & $\begin{array}{c}\mathrm{H} \alpha \text { Flux }^{\mathrm{a}} \\
\left(10^{-13} \mathrm{erg} \mathrm{s}^{-1} \mathrm{~cm}^{-2}\right)\end{array}$ & $\begin{array}{c}\text { Fraction of } \\
\text { Total Flux }\end{array}$ \\
\hline Outer N arm & 38.7 & $37 \%$ \\
Inner N arm & 23.0 & $22 \%$ \\
Inner S arm & 23.0 & $22 \%$ \\
Outer S arm & 9.4 & $9 \%$ \\
Nucleus & 4.7 & $4.5 \%$ \\
Body & 6.3 & $6 \%$ \\
Detached Clump & 0.31 & $0.3 \%$ \\
\hline
\end{tabular}

${ }^{a}$ using the total flux calibration of Dopita \& Ryder 1994. 
This figure "Fig01.jpg" is available in "jpg" format from: http://arxiv.org/ps/astro-ph/9701015v1 
This figure "Fig02.jpg" is available in "jpg" format from: http://arxiv.org/ps/astro-ph/9701015v1 
This figure "Fig03.gif" is available in "gif" format from: http://arxiv.org/ps/astro-ph/9701015v1 
This figure "Fig04.gif" is available in "gif" format from: http://arxiv.org/ps/astro-ph/9701015v1 
This figure "Fig05.gif" is available in "gif" format from: http://arxiv.org/ps/astro-ph/9701015v1 
This figure "Fig06.gif" is available in "gif" format from: http://arxiv.org/ps/astro-ph/9701015v1 
This figure "Fig07.gif" is available in "gif" format from: http://arxiv.org/ps/astro-ph/9701015v1 
This figure "Fig08.gif" is available in "gif" format from: http://arxiv.org/ps/astro-ph/9701015v1 
This figure "Fig09.gif" is available in "gif" format from: http://arxiv.org/ps/astro-ph/9701015v1 
This figure "Fig10.gif" is available in "gif" format from: http://arxiv.org/ps/astro-ph/9701015v1 
This figure "Fig11.gif" is available in "gif" format from: http://arxiv.org/ps/astro-ph/9701015v1 